\title{
INTEGRABILITY CONDITIONS OF A RESONANT SADDLE IN GENERALIZED LIÉNARD-LIKE COMPLEX POLYNOMIAL DIFFERENTIAL SYSTEMS
}

\author{
JAUME GINÉ ${ }^{1}$ AND JAUME LLIBRE ${ }^{2}$
}

\begin{abstract}
We consider a complex differential system with a resonant saddle at the origin. We compute the resonant saddle quantities and using Gröbner bases we find the integrability conditions for such systems up to a certain degree. We also establish a conjecture about the integrability conditions for such systems when they have arbitrary degree.
\end{abstract}

\section{INTRODUCTION}

The center problem for polynomial vector fields in the real plane with an elementary singular point of the form

$$
\dot{x}=-y+\cdots, \quad \dot{y}=x+\cdots,
$$

where the dots means higher order terms is a subject of much work during these last decades, see for instance $[2,6]$. These type of systems can be embedded by the change of variable $u=x+i y$ and the corresponding conjugate variable $v=x-i y$ into the complex system of the form

$$
\dot{u}=u+\cdots, \quad \dot{v}=-v+\cdots .
$$

The next generalization of the above system is to consider the case of a polynomial system in the complex plane of the form

$$
\dot{u}=\lambda_{1} u+\cdots, \quad \dot{v}=-\lambda_{2} v+\cdots,
$$

where $\lambda_{1}, \lambda_{2} \in \mathbb{C}$. However if $k:=\left(\lambda_{1}, \lambda_{2}\right)$ does not satisfies the resonant condition $(\alpha, k)-\lambda_{m}=0$, for all $m \in\{1,2\}$ and for all $\alpha \in \mathbb{N}_{0}^{2}$ with $|\alpha| \geq 2$ then system (1) is formally equivalent to its normal form $\dot{u}=\lambda_{1} u, \dot{v}=-\lambda_{2} v$, see [6]. Hence we consider the case with a $p:-q$ resonant elementary singular point

$$
\dot{u}=p u+\cdots, \quad \dot{v}=-q v+\cdots,
$$

2010 Mathematics Subject Classification. Primary 34C05. Secondary 37C10.

Key words and phrases. center problem, analytic integrability, polynomial complex differential systems. 
with $p, q \in \mathbb{Z}$. If $p, q>0,(p, q)=1$ then the linear part has the analytic first integral $H_{0}=x^{q} y^{p}$ and we can seek the conditions for the existence of an analytic first integral $H=H_{0}+\cdots$ for system (2). Hence get the equation $\dot{H}=v_{1} H_{0}^{2}+v_{3} H_{0}^{3}+\cdots$, and the so-called $p:-q$ resonant saddle quantities $v_{i}$ are polynomials in the coefficients of system (2). If all the $v_{i}$ are zero we say that we have an analytic resonant saddle, see [7] and references therein.

In this work we aim to study analytic differential systems in the complex plane of the form

$$
\dot{x}=x+g(x) y, \quad \dot{y}=-y+f(y) x,
$$

where $f(y)=\sum_{j \geq 1} a_{j} y^{j}$ and $g(x)=\sum_{j \geq 1} b_{j} x^{j}$ are analytic functions without constant terms. In fact system (3) has a $1:-1$ resonant saddle singular point at the origin. System (3) with $g(x)=0$ was studied in [4] where the following result was given.

Theorem 1. ([4]) The complex polynomial differential system (3) with $g(x)=0$ has an integrable saddle at the origin if and only if one of the following two conditions holds:

(1) $a_{1}=a_{2}=0$;

(2) $a_{i}=0$ for $i \geq 2$.

The case $g(x) \neq 0$ is much harder and we have studied the polynomial case when $f$ and $g$ are polynomials of degree less than or equal to 6 , and we have obtained the following result.

Theorem 2. The complex polynomial differential system (3) when $f$ and $g$ are polynomials of degree $\leq 6$ has an analytic integrable saddle at the origin, if and only if, the following conditions hold:

$a_{2}+b_{2}=-a_{1} a_{3}+b_{1} b_{3}=a_{1}^{2} a_{4}+b_{1}^{2} b_{4}=-a_{1}^{3} a_{5}+b_{1}^{3} b_{5}=a_{1}^{4} a_{6}+b_{1}^{4} b_{6}=0$.

The proof of this theorem is given in section 2 .

From this result we can establish the following conjecture for the complex polynomial differential system (3) when the degrees of the polynomials $f$ and $g$ are arbitrary.

Conjecture 3. The complex polynomial differential system (3) has an analytic integrable saddle at the origin, if and only if, the following conditions holds:

$$
\begin{aligned}
a_{2}+b_{2} & =0, \\
-a_{1}^{i-2} a_{i}+b_{1}^{i-2} b_{i} & =0, \text { for } i \text { odd } \\
a_{1}^{i-2} a_{i}+b_{1}^{i-2} b_{i} & =0, \text { for } i \text { even } .
\end{aligned}
$$




\section{Proof of Theorem 2}

The sufficiency of Theorem 2 is proved in the following lemma.

Lemma 4. System (3) when $f$ and $g$ are polynomials of degree $\leq 6$ with $a_{2}+b_{2}=-a_{1} a_{3}+b_{1} b_{3}=a_{1}^{2} a_{4}+b_{1}^{2} b_{4}=-a_{1}^{3} a_{5}+b_{1}^{3} b_{5}=a_{1}^{4} a_{6}+\bar{b}_{1}^{4} b_{6}=0$ has an analytic first integral defined in a neighborhood of the origin.

Proof. First we vanish the conditions taking $b_{2}=-a_{2}, a_{3}=k_{3} b_{1}$, $b_{3}=k_{3} a_{1}, a_{4}=-k_{4} b_{1}^{2}, b_{4}=k_{4} a_{1}^{2}, a_{5}=k_{5} b_{1}^{3}, b_{5}=k_{5} a_{1}^{3}, a_{6}=-k_{6} b_{1}^{4}$, $b_{6}=k_{6} a_{1}^{4}$, where $k_{3}, k_{4}, k_{5}$ and $k_{6}$ are arbitrary constants. System (3) takes the form

$$
\begin{gathered}
\dot{x}=x+\left(a_{1} x+a_{2} x^{2}+b_{1} k_{3} x^{3}-b_{1}^{2} k_{4} x^{4}+b_{1}^{3} k_{5} x^{5}-b_{1}^{4} k_{6} x^{6}\right) y, \\
\dot{y}=-y+\left(b_{1} y-a_{2} y^{2}+a_{1} k_{3} y^{3}+a_{1}^{2} k_{4} y^{4}+a_{1}^{3} k_{5} y^{5}+a_{1}^{4} k_{6} y^{6}\right) x .
\end{gathered}
$$

Now we do the change of coordinates $X=b_{1} x$ and $Y=a_{1} y$ and the scaling of time $d t=d \tau /\left(b_{1} a_{1}\right)$ and the system is transformed into

$$
\begin{aligned}
& \dot{X}=X\left(b_{1} a_{1}+Y\left(b_{1} a_{1}+a_{2} X+k_{3} X^{2}-k_{4} X^{3}+k_{5} X^{4}-k_{6} X^{5}\right)\right), \\
& \dot{Y}=Y\left(-b_{1} a_{1}+X\left(b_{1} a_{1}-a_{2} Y+k_{3} Y^{2}+k_{4} Y^{3}+k_{5} Y^{4}+k_{6} Y^{5}\right)\right) .
\end{aligned}
$$

Finally we do a rotation of angle $\varphi=\pi / 4$ which is given by the linear change

$$
u=\frac{X}{\sqrt{2}}-\frac{Y}{\sqrt{2}}, \quad v=\frac{X}{\sqrt{2}}+\frac{Y}{\sqrt{2}},
$$

and the system takes the form

$$
\dot{u}=v+v P\left(u, v^{2}\right), \quad \dot{v}=u+Q\left(u, v^{2}\right) .
$$

System (4) is invariant by the symmetry $(u, v, t) \rightarrow(u,-v,-t)$. hence the system is a time-reversible system following the definition given in [5]. Moreover all the time-reversible systems are inside the Sibirsky subvariety which are inside the Center variety i.e., systems (4) that have an analytic first of the form $H=u v+\cdots$ around the origin. Hence system (4) and the original one have a resonant integrable saddle at the origin.

The necessity condition of Theorem 2 is proved in the following lemma.

Lemma 5. If system (3) when $f$ and $g$ are of degree $\leq 6$ has an integrable saddle at the origin then the following conditions hold:

$a_{2}+b_{2}=-a_{1} a_{3}+b_{1} b_{3}=a_{1}^{2} a_{4}+b_{1}^{2} b_{4}=-a_{1}^{3} a_{5}+b_{1}^{3} b_{5}=a_{1}^{4} a_{6}+b_{1}^{4} b_{6}=0$. 
Proof. In system (3) with $f$ and $g$ polynomials of degree $\leq 6$ we introduce the change of variables $X=x+i y, Y=x-i y$ and the scaling of time $t \mapsto-t / i$. With this change the system becomes into the form

$$
\begin{aligned}
\dot{X} & =-Y+F(X, Y), \\
\dot{Y} & =X+G(X, Y),
\end{aligned}
$$

where the coefficients of $F$ and $G$ are complex. Next we take polar coordinates, i.e., $X=r \cos \theta$ and $Y=r \sin \theta$ and doing this change of variables system (5) takes the form

$$
\dot{r}=\sum_{s=2}^{7} P_{s}(\theta) r^{s}, \quad \dot{\theta}=1+\sum_{s=2}^{7} Q_{s}(\theta) r^{s-1},
$$

where $P_{s}$ and $Q_{s}$ are trigonometric polynomials of degree $s$. To determine the necessary conditions to have a formal first integral in a neighborhood of the origin we propose a Poincaré series of the form $H(r, \theta)=\sum_{m=2}^{\infty} H_{m}(\theta) r^{m}$, where $H_{2}(\theta)=1 / 2$ and $H_{m}(\theta)$ are homogeneous trigonometric polynomials respect to $\theta$ of degree $m$. Imposing that this power series is a formal first integral of system (6) we obtain $\dot{H}(r, \theta)=\sum_{k=2}^{\infty} V_{2 k} r^{2 k}$, where the $V_{2 k}$ are in fact the saddle quantities that depend on the parameters of system (3). From the recursive equations that generate $V_{2 k}$ we can see that these $V_{2 k}$ are polynomials in the parameters of system (3), see $[1,3,6]$. Due to the Hilbert Basis theorem, the ideal $J=<V_{4}, V_{6}, \ldots>$ generated by the saddle quantities is finitely generated, i.e. there exist $v_{1}, v_{2}, \ldots, v_{k}$ in $J$ such that $J=<v_{1}, v_{2}, \ldots, v_{k}>$. Such a set of generators is called a basis of $J$ and the conditions $v_{j}=0$ for $j=1, \ldots, k$ provide a finite set of necessary conditions to have a formal first integral around the origin.

In fact we determine a number of saddle quantities thinking that inside these number there is the set of generators.

In our case the necessity is straightforward because the first saddle quantity is $V_{4}=a_{2}+b_{2}$. Then we take $b_{2}=-a_{2}$. The next saddle quantity is $V_{6}=a_{1} a_{3}-b_{1} b_{3}$. To vanish this quantity we take $a_{3}=k_{3} b_{1}$ and $b_{3}=k_{3} a_{1}$ where $k_{3}$ is an arbitrary constant. We compute the next saddle quantity and we obtain $V_{8}=a_{1}^{2} a_{4}+b_{1}^{2} b_{4}$. Hence we take, as before, $a_{4}=k_{4} b_{1}^{2}$ and $b_{4}=-k_{4} a_{1}^{2}$, where $k_{4}$ is an arbitrary constant. The next saddle quantity is $V_{10}=a_{1}^{3} a_{5}-b_{1}^{3} b_{5}$. Hence we take, as before, $a_{5}=k_{5} b_{1}^{3}$ and $b_{5}=k_{5} a_{1}^{3}$, where $k_{5}$ is an arbitrary constant. Under these conditions $V_{12}=a_{1}^{4} a_{6}+b_{1}^{4} b_{6}$ and we take $a_{6}=k_{6} b_{1}^{4}$ and $b_{6}=-k_{6} a_{1}^{4}$, where $k_{6}$ is an arbitrary constant. The next saddle quantity $V_{14}$ is zero and we assume that the rest are also zero and that we have vanished a set of generators. 
Proof of Theorem 2. The proof of Theorem 2 is an immediate consequence of Lemmas 4 and 5.

\section{ACKnowledgements}

The first author is partially supported by a MINECO/ FEDER grant number MTM2014-53703-P and an AGAUR (Generalitat de Catalunya) grant number 2014SGR 1204. The second author is partially supported by a MINECO grant MTM2013-40998-P, an AGAUR grant number 2014SGR-568, and the grants FP7-PEOPLE-2012-IRSES 318999 and 316338 .

\section{REFERENCES}

[1] B. Ferčec, J. Giné, V.G. Romanovski, V.F. Edneral, Integrability of complex planar systems with homogeneous nonlinearities, J. Math. Anal. Appl. 434 (2016), no. 1, 894-914.

[2] H. Giacomini, J. Giné And J. Llibre, The problem of distinguishing between a center and a focus for nilpotent and degenerate analytic systems, J. Differential Equations 227 (2006), 406-426.

[3] J. Giné, C. VAlls, Integrability conditions of a resonant saddle perturbed with homogeneous quintic nonlinearities, Nonlinear Dynam. 81 (2015), no. 4, 2021-2030.

[4] J. Giné, C. VALLs, Integrability conditions of a resonant saddle in Liénardlike complex systems, Chaos, Solitons and Fractals 82 (2016), 139-141.

[5] A.S. Jarrah, R. Laubenbacher, V.G. Romanovski, The Sibirsky component of the center variety of polynomial differential systems, J. Symbolic Comput. 35 (2003), no. 5, 577-589.

[6] V.G. Romanovski, D.S. Shafer, The Center and Cyclicity Problems: A Computational Algebra Approach, Birkhäuser, Boston, 2009.

[7] QI Zhang, Y. LiU, Integrability and generalized center problem of resonant singular point, Appl. Math. Lett. 40 (2015), 13-16.

2 Departament de Matemàtica, Universitat de Lleida, Avda. Jaume II, 69; 25001 Lleida, CAtalonia, Spain

E-mail address: gine@matematica.udl.cat

${ }^{1}$ Departament de Matemàtiques, Universitat Autònoma de Barcelona, 08193 Bellaterra, Barcelona, Catalonia, Spain

E-mail address: 1libre@mat.uab.cat 